\title{
Unzureichende Datenlage für eine Änderung der Praxis
}

\author{
Ursula Winterfelda, David Baud ${ }^{\mathrm{b}}$, Alice Panchaud ${ }^{\mathrm{a}}$, Laura Rothuizen ${ }^{\mathrm{a}}$, Françoise Livio ${ }^{\mathrm{a}}$, Rudolf Stollerc, \\ Thierry Buclina \\ a Swiss Teratogen Information Service, Division de Pharmacologie clinique, CHUV, Lausanne; ${ }^{b}$ Unité de recherche en Médecine materno-foetale, \\ Département de Gynécologie-Obstétrique, Maternité, CHUV, Lausanne; ${ }^{c}$ Swissmedic, Schweizerisches Heilmittelinstitut, Bern
}

\begin{abstract}
Im Jahr 2014 wurde in den Medien ein möglicher Zusammenhang zwischen der Einnahme von Paracetamol während der Schwangerschaft und dem Auftreten von psychomotorischen Entwicklungsstörungen und Aufmerksamkeitsdefizit-Hyperaktivitätsstörung (ADHS) bei Kindern diskutiert. Die Ergebnisse der Studien haben sich als unzureichend erwiesen, um dieses Risiko zu belegen. Der therapeutische Stellenwert von Paracetamol in der Schwangerschaft ist dadurch nicht in Frage gestellt.
\end{abstract}

\begin{abstract}
Mehrere neuere Studien haben die Einnahme von $\mathrm{Pa}$ racetamol in der Schwangerschaft mit einem erhöhten Risiko von psychomotorischen Entwicklungsstörungen und ADHS in Verbindung gebracht.Da die Ergebnisse dieser Studien weithin von den Medien übernommen und in alarmierenden Artikeln verbreitet wurden, kam es wahrscheinlich zu einer übertriebenen Risikowahrnehmung sowohl bei den Fachleuten als auch bei den Patientinnen.
\end{abstract}

\section{Interessantes Teratovigilance-Signal - Nachweis des Kausalzusammenhangs fehlt}

Streissguth AP, Treder RP, Barr HM, Shepard TH, Bleyer WA, Sampson PD, Martin DC. Aspirin and acetaminophen use by pregnant women and subsequent child IQ and attention decrements. Teratology. 1987;35:211-9.

2 Brandlistuen RE, Ystrom E, Nulman I, Koren $\mathrm{G}$, Nordeng $\mathrm{H}$ Prenatal paracetamol exposure and child neurodevelopment: a sibling-controlled cohort study. Int $\mathrm{J}$ Epidemiol. 2013;42:1702-13.
Eine erste prospektive Kohortenstudie von 1987 hat keine Auswirkungen einer Paracetamol-Behandlung während der Schwangerschaft auf Intelligenzquotient oder Aufmerksamkeit von Kindern bis zum Alter von vier Jahren beobachtet [1]. Es handelte sich dabei um eine Kohorte von 1529 schwangeren Frauen, von denen 41\% Paracetamol während der ersten Hälfte der Schwangerschaft eingenommen hatten. Drei Studien, die zwischen 2013 und 2014 veröffentlicht wurden, haben jedoch einen Zusammenhang zwischen einer Paracetamol-Einnahme in der Schwangerschaft und psychomotorischen Entwicklungsstörungen oder ADHS der in utero exponierten Kinder beschrieben [2-4].
In einer norwegischen Kohortenstudie mit 48631 schwangeren Frauen wurden psychomotorische Entwicklungsunterschiede zwischen 2919 Geschwistern untersucht, von denen jeweils nur eines in utero exponiert war [2]. Die Mütter haben die psychomotorische Entwicklung ihrer Kinder im Alter von drei Jahren mittels verschiedenen Fragebögen und validierten Skalen beurteilt. Die Einnahme von Paracetamol für eine Dauer von 28 Tagen oder mehr während der Schwangerschaft war mit einem erhöhten Risiko für eine beeinträchtigte psychomotorische Entwicklung einschliesslich der motorischen Fähigkeiten, der Kommunikation, des Verhaltens und der Aktivität der exponierten Kinder verbunden. Bei einer Prävalenz von ADHS und psychomotorischen Störungen in der allgemeinen pädiatrischen Population von $6 \%$ entsprechen die Ergebnisse dieser Studie einem relativen Risiko von 1,7 oder einem absoluten Risiko von $10 \%$. Eine Behandlungsdauer von weniger als 28 Tagen während der Schwangerschaft ergab ebenfalls negative Auswirkungen auf die motorischen Fähigkeiten der exponierten Kinder, es wurden jedoch keine Auswirkungen auf alle anderen untersuchten Parameter beobachtet.

Eine dänische Kohortenstudie mit 64322 Kindern untersuchte den Zusammenhang zwischen einer Anwendung von Paracetamol in der Schwangerschaft und dem Risiko für ADHS der exponierten Kinder. Mehr als die Hälfte der Mütter gab an, während der Schwangerschaft Paracetamol eingenommen zu haben. Folgende Kriterien wurden bei der Auswertung berücksichtigt: elterliche Beurteilung des Verhaltens ihrer Kinder im Alter von sieben Jahren (validierter Fragebogen); 
ADHS-Diagnose in einem der beiden untersuchten Gesundheitsregister; Arzneimitteltherapie für ADHS in einem Verschreibungsregister. Mehrere Störfaktoren wurden berücksichtigt (Infektion oder entzündliche Erkrankung während der Schwangerschaft, psychische Gesundheit der Mutter). Die Einnahme von Paracetamol ging mit einem erhöhten Risiko für hyperaktives Verhalten im Alter von sieben Jahren (relatives Risiko oder risk ratio 1,13, 95\% Konfidenzintervall (KI) 1,01 bis 1,27), einer ADHS-Diagnose (hazard ratio [HR] 1,37, 95\% KI 1,19 bis 1,59) und einer Behandlung von ADHS mit einem rezeptpflichtigen Medikament (HR 1,29, 95\% KI 1,15 bis 1,44) einher [3]. Die Assoziation war bei längerer Behandlungsdauer stärker.

\section{Paracetamol bleibt das Analgetikum der Wahl während der Schwangerschaft.}

Eine neuseeländische Kohortenstudie mit 871 Kindern hat den Zusammenhang zwischen verschiedenen Arzneimittelanwendungen in der Schwangerschaft (Paracetamol, Aspirin, Antazida, Antibiotika) und dem Auftreten von Verhaltensstörungen oder ADHS-Symptomen bei Kindern im Alter von sieben und elf Jahren untersucht [4]. Die Autoren haben eine Reihe von Störfaktoren (Geburtsgewicht, sozioökonomischer Status, mütterlicher Stress) berücksichtigt. Die Beurteilung des Verhaltens der Kinder wurde von den Eltern und den Kindern selbst mit Hilfe von Fragebögen und validierten Skalen durchgeführt. Fast die Hälfte (49,8\%) der Mütter gab an, Paracetamol während der Schwangerschaft eingenommen zu haben. Auf einer Skala von 40 Punkten schnitten die in utero exponierten Kinder etwa 1 Punkt schlechter ab als die nicht exponierten Kinder.

\section{Vorsicht bei der Interpretation der Ergebnisse}

Mehrere Faktoren müssen bei der Interpretation der Ergebnisse dieser Studien berücksichtigt werden. Die in den beiden skandinavischen Studien beobachteten Ergebnisse lagen an der Grenze der statistischen Signifikanz, obwohl Tausende von Patienten berücksichtigt wurden. Die Ergebnisse der neuseeländischen Studie waren ebenfalls von geringer klinischer Relevanz. Eine Vielzahl der Ergebnisse basiert auf der elterlichen Beurteilung des Verhaltens ihrer Kinder mittels unterschiedlicher Fragebögen und Skalen, ohne dass etwaige Diagnosen medizinisch bestätigt wurden. In beiden skandinavischen Studien waren die Assoziationen bei längerer Therapiedauer (>28 Tage, > ein Trimenon) stärker ausgeprägt. Eine solche Anwendung von Para- cetamol während der Schwangerschaft ist ungewöhnlich, und es kann nicht ausgeschlossen werden, dass eine Erkrankung der Mutter bei den beobachteten Ergebnissen eine Rolle gespielt hat. Psychomotorische Entwicklungsstörungen und ADHS sind multifaktoriellen Ursprungs. Obwohl einige Störfaktoren in den statistischen Analysen berücksichtigt wurden, könnten potentielle Störfaktoren (somatische oder psychische Erkrankungen der Mutter, Konsum von Alkohol oder illegalen Substanzen während der Schwangerschaft, Umweltfaktoren in den ersten Lebensjahren der Kinder usw.) sowohl mit der Paracetamol-Einnahme der Mutter während der Schwangerschaft als auch mit dem Auftreten von ADHS bei den Kindern assoziiert sein. Da die tatsächlich eingenommene ParacetamolDosis nicht bekannt war, gibt es Zweifel bezüglich des tatsächlichen Umfangs der mütterlichen Exposition sowie der Interpretation eines Dosis-Wirkungs-Effekts. Insgesamt haben diese Studien eine schwache Assoziation zwischen einer Anwendung von Paracetamol in der Schwangerschaft und dem Risiko von psychomotorischen Entwicklungsstörungen und ADHS bei den exponierten Kindern beobachtet, ohne einen Nachweis für einen Kausalzusammenhang zu liefern.

\section{Stellenwert von Paracetamol in der Schwangerschaft nicht in Frage gestellt}

Die vor kurzem veröffentlichten Beobachtungen sind unzureichend, um eine indikationsgerechte Anwendung von Paracetamol in Frage zu stellen, und dieses Arzneimittel bleibt das Analgetikum der Wahl während der Schwangerschaft. Therapeutische Alternativen wie NSAIDs haben ein weniger günstiges Sicherheitsprofil als Paracetamol. Weiterhin rechtfertigen die neuen Daten den Austausch von Paracetamol mit Opioiden zur Behandlung leichter bis mittelschwerer Schmerzen während der Schwangerschaft nicht. Es ist jedoch so gut wie niemals möglich, jegliches Risiko einer Arzneimitteltherapie in der Schwangerschaft formell auszuschliessen. Dies gilt insbesondere in Situationen, in denen ein Arzneimittel ausserhalb des Kontextes eingenommen wird, in dem die Mehrzahl der Sicherheitsdaten in der Schwangerschaft erhoben wurden, wie beispielsweise bei der Einnahme von supratherapeutischen Dosen oder einer länger als üblichen Behandlungsdauer. Daher sollten schwangere Frauen grundsätzlich Arzneimittel nur indikationsgerecht anwenden.

Wir möchten daran erinnern, dass unerwünschte Arzneimittelwirkungen (UAW) vorzugsweise über das Online-Portal ElViS oder mittels des gelben Formulars zu melden sind, siehe www.swissmedic.ch 